\title{
Microcosme, Macrocosme
}

\section{Microcosm, Macrocosm}

\author{
Jean Girel ${ }^{1}$ \\ Artiste, gireljean@orange.fr
}

RÉSUMÉ. Le sujet de mon exposition actuelle à la galerie Arcanes à Paris est une fois de plus la nature, dans son immensité avec les paysages, dans son intimité avec les thèmes animaliers ou les épidermes de fruits. Les outils utilisés pour exprimer ces thèmes sont les phénomènes céramiques de fusion, de cristallisation, d'ébullition ou de tension superficielle.

ABSTRACT. The subject of my current exhibition at the Arcanes gallery in Paris is once again nature, in its immensity with landscapes, in its intimacy with animal themes or fruit epidermis. The tools used to express these themes are the ceramic phenomena of fusion, crystallization, boiling or surface tension.

MOTS-CLÉS. ceramique, porcelaine, nature, matière, phénomènes.

KEYWORDS. ceramic, porcelain, nature, matter, phenomena.

J'avais profité de la période morose où toutes les expositions étaient reportées ou supprimées pour me consacrer uniquement à la recherche de l'irisation de couvertes noires « yohen temmoku », mais lorsqu'une éclaircie est survenue, j'ai ressenti un besoin de couleur, de fraîcheur, de gaité et me suis replongé dans la transposition du miracle de la nature qui m'environne en tentant d'en exprimer l'étendue, la fusion de la terre et du ciel dans une série de disques et de seaux; en me penchant au dessus de la mare pour observer la ronde des grenouilles, et en faisant tourner entre mes mains les légumes, les fruits du jardin et ceux, plus exotiques que notre voisine nous apporte dès qu'elle en reçoit un arrivage pour transcrire leur texture, leur toucher et les bizarreries que le hasard et la nécessité leur impose. Ce renouveau nous a permis avec la galerie Arcanes de fixer une date d'exposition à Paris.

Pratiquant ce que je nomme «une céramique des phénomènes », j'ai dans la série des paysages joué avec la fluidité ou à l'inverse la minéralité de la matière pour exprimer le ciel, le terre et leur rencontre.

Dans la série des boîtes grenouilles, c'est le contrôle de la micro-cristallisation qui m'a permis de trouver le caractère embué de leur épiderme ; pour les coupes tortues, c'est entre autres le broyage de la couverte brune à un stade colloïdal qui a su entrainer au séchage des retirements de matière brune sur un fond jaune évoquant leur carapace.

Quant à la série des vases et coupelles fruits, j'ai joué sur les décollements de matière, sur la franche ébullition du fer ou le discret pétillement du manganèse pour développer une infinité de textures, de surfaces «peau d'orange » ou écorce, l'ensemble des pièces étant cuits à 1300/1340 en atmosphère franchement oxydant pour amener les couleurs à leur intensité maximale.

Sites :

Galerie Arcanes

11 rue Bonaparte, Paris $6^{\mathrm{e}}$

galeriearcanes.fr

Exposition du 9 au 30 décembre 2021 Mardi-Samedi 11h-13h / 14h-19h 


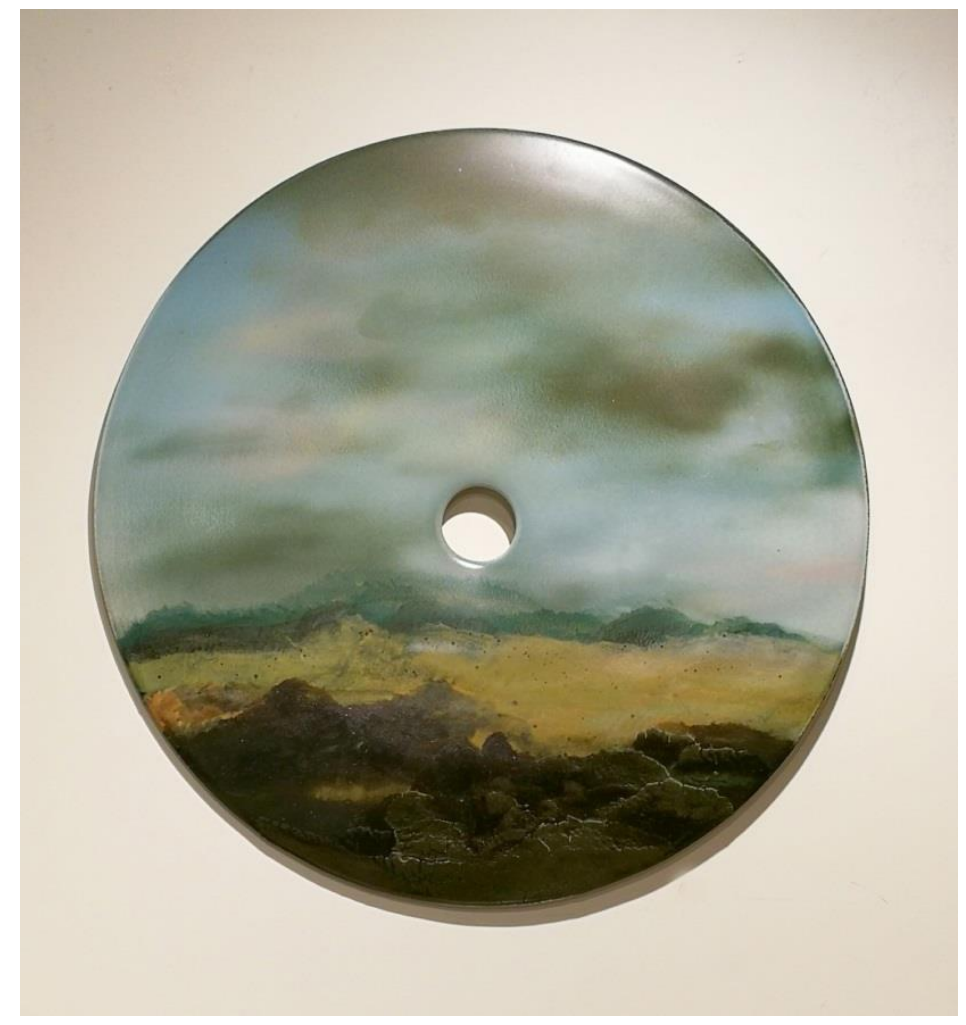

Disque « Bi », paysage (diamètre : $47 \mathrm{~cm}$ )

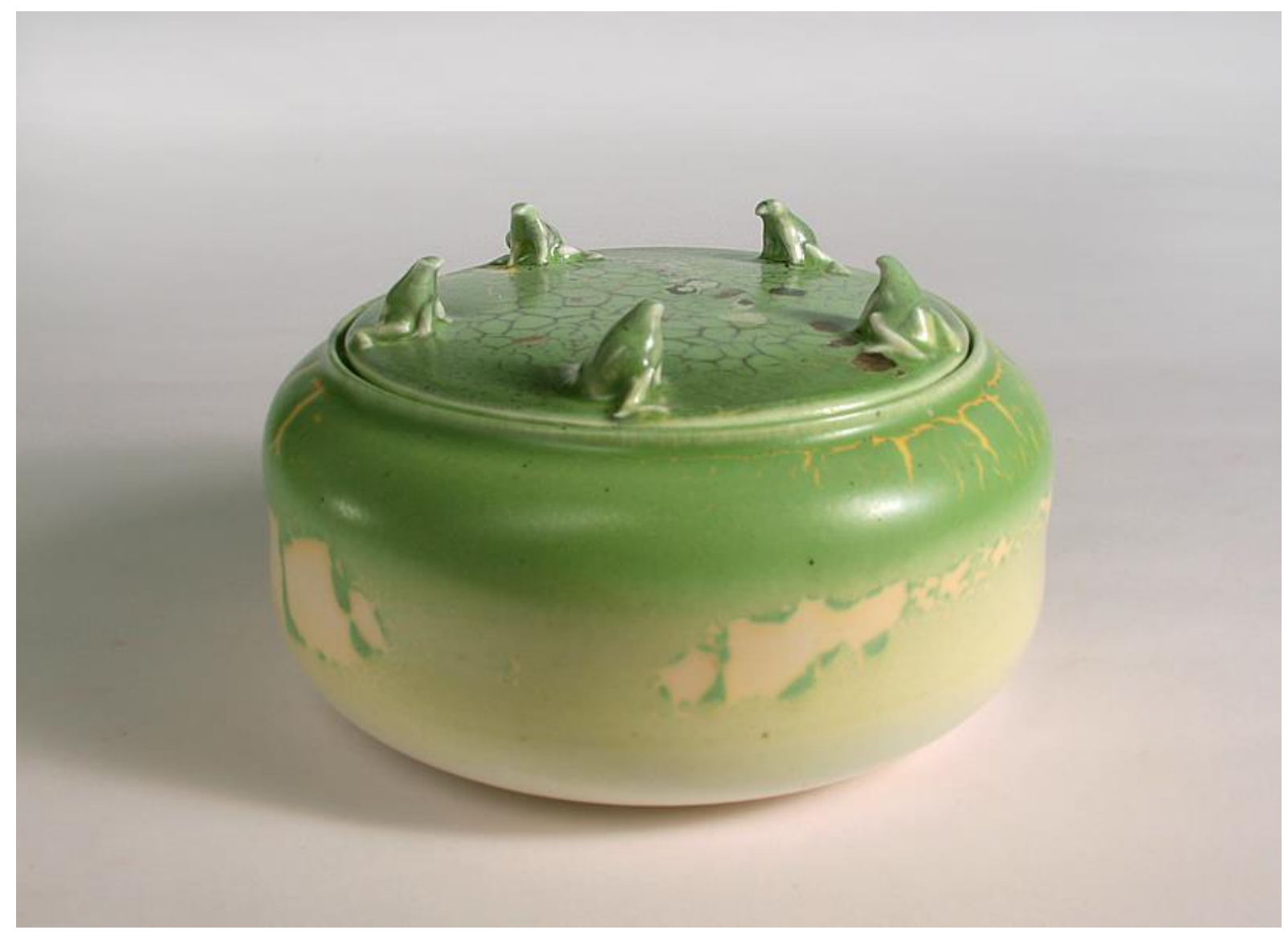

Boîte grenouille (diamètre : $27 \mathrm{~cm}$ ) 


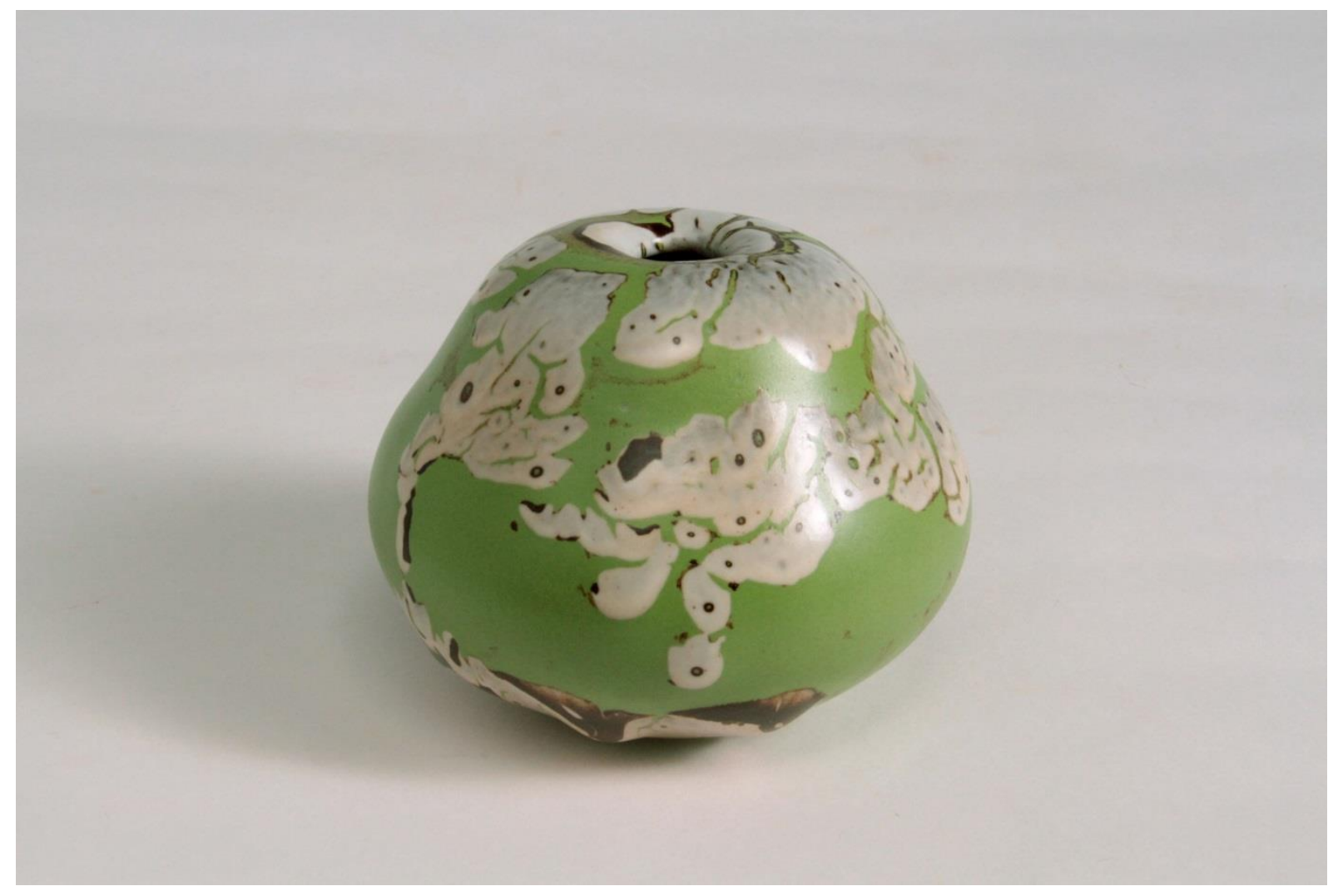

Vase fruit (hauteur : $11 \mathrm{~cm}$ ) 